\title{
LEARNING THE ROPES: RAAS: MEASURING ITS COMPONENTS AND NEW THERAPEUTIC STRATEGIES Introduction
}

\author{
THOMAS UNGER \\ CARIM - Maastricht University \\ Maastricht, The Netherlands
}

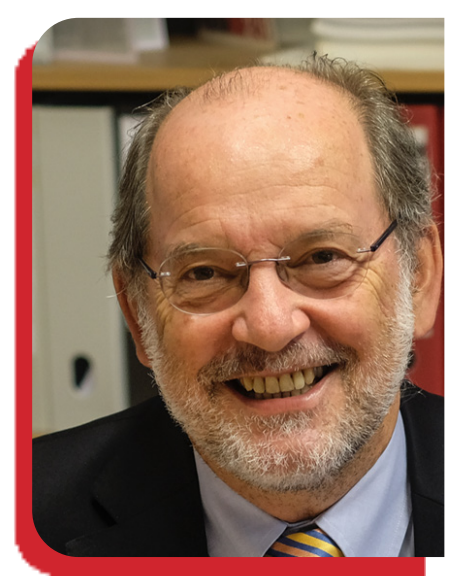

DOI: $10.30824 / 2112-4$

The renin-angiotensin-aldosterone system (RAAS) has inspired cardiovascular research since the discovery of renin by Tigerstedt and Bergmann in 1898'. They reported that an extract from rabbit kidney would raise blood pressure when injected into a recipient rabbit. In the decades to follow, the individual RAAS components: the precursor protein angiotensinogen, the enzyme renin, the converting enzymes ACE and ACE2, the various angiotensin peptides, notably the octapeptide, angiotensin II (Ang II), as well as the adrenal steroid aldosterone with their different receptors were stepwise identified, and methods to analyze, measure and monitor all these components were developed over the years around the world. Today, we can fill libraries with literature, not only about presence, regulation, and concentration of the individual proteins, peptides and of aldosterone in blood and various tissues of many species, but also about their interplay with other relevant hormonal systems and many aspects of their physiological and pathophysiological functions.

Concerning hypertension, an inadequately regulated RAAS has been identified as a major player contributing to the induction and maintenance of the disease: Ang II, a prominent effector peptide of the renin-angiotensin system (RAS), raises via its AT1 receptor blood pressure by a direct vasopressor effect. In addition, it can also exert a plethora of further actions in the kidney and other organs contributing not only to the increase of blood pressure but also, among others, to vascular and cardiac hypertrophy, fibrosis, inflammation, or a decrease of insulin sensitivity. Similar actions have been ascribed to aldosterone, which is to a certain part under the control of Ang II.

In view of these unwanted pathological features of the RAAS, it is quite understandable that laboratories in the pharmaceutical industry as well as in academia, often in close collaboration, were driven by the idea that an inhibition of the system could be beneficial in lowering elevated blood pressure and by this preventing or reducing the sequelae of hypertension such as stroke, renal and cardiac failure. The first aldosterone antagonist, spironolactone, was brought to the market in the sixties of the last century, followed by the converting enzyme inhibitors in the eighties and the angiotensin AT1 receptor antagonists in the nineties. These newly established drug classes had a tremendous impact on the management of cardio-metabolic diseases reaching far beyond the mere reduction of blood pressure, and today's drug armamentarium is unthinkable without compounds inhibiting the RAAS.

However, despite this unquestionably huge progress in our knowledge of the RAAS and its manipulation on a molecular and clinical level, it appears that with each step of advancement new questions arise and new therapeutic options are unveiled. A good example for this is the discovery of the ACE2-angiotensin (1-7) branch of the RAS or the angiotensin AT2 receptor whose tissueprotecting actions may become therapeutic tools in the future ${ }^{2,3}$.

In the current 'Learning the Ropes' section you will find four articles on RAAS measurement methodology and novel therapeutic perspectives written by prominent experts in the field.

Joel Ménard, a pioneer and doyen of RAAS research, opens the section with an article of how to measure renin and where renin measurements can be used clinically. For quite a time, renin was the only measurable compound of the RAS in the blood, and classifications of hypertension (low-, normal-, high renin hypertension) were based on this information. In a historical perspective, Joel Ménard describes the long 
and cumbersome way of how these measurements were begun with a relative crude bioassay methodology and were then continuously refined over the years towards the laboratory routine of our days.

Jumping right into our current days, Marko Poglitsch describes a novel method he and his colleagues have developed to simultaneously measure various angiotensin peptides and aldosterone in the blood together with angiotensin-based calculation of RAS proteins like renin and ACE. For the first time, this methodology provides an exact pattern of RAAS components under different clinical and therapeutical circumstances. It allows not only for the identification of hypertension subtypes but also for the demonstration of possible causes of therapeutic failure i.e. uncontrolled hypertension. This approach may open up new perspectives towards an individualized, more successful, drug therapy.

Reure Lopez, Edward Sturrock and Rhian Touyz present us with a new therapeutic option, the ACE-Cdomain-neprilysin inhibitor. The idea to combine ACE inhibition with neprilysin inhibition in one molecule was first entertained by the development of the compound omapatrilat. However, due to a doubling of angioedemea, a well-known, potentially dangerous, side effect of ACE inhibitors, this compound never entered the market. More recently, another pharmaceutical company has developed a supramolecular salt complex of an AT1-blocker and a neprilysin inhibitor (ARNI). This compound has proven to be very successful, mainly in the treatment of heart failure with low ejection fraction. Rhian Touyz and colleagues have chosen a different approach: They inhibit the C-domain of ACE with a compound called lisW-S and combine it with the neprilysin inhibitor sacubitril. By this, they only lower the production of Ang II with no effects on bradykinin and other peptides, thus avoiding the ACE inhibitorinduced bradykinin accumulation which is thought to be a mechanism of cough and angioedema formation. The authors demonstrate that, in animal experiments, the combination of lisW-S+sacubitril lowers blood pressure like omapatrilat but does not lead to a bradykinin accumulation.

Finally, Liwei Ren and Jan Danser surprise us with a completely new molecular approach to inhibit the RAAS by targeting angiotensinogen, the precursor of all angiotensin peptides, via interfering RNA or antisense oligonucleotides (ASO). They describe experiments with a siRNA in genetically hypertensive rats (SHR) or in a low renin model of chronic kidney disease (CKD). In SHR, they could decrease blood pressure to the same extent as with classical RAS inhibitors with the advantage of a much lower dosing frequency. Combination with an AT1 receptor blocker augmented the blood pressure lowering effects. In CKD, they observed tissue protecting effects with a moderate reduction of blood pressure.

These four examples may suffice to demonstrate that the venerable RAAS still represents a rich source of inspiration to creative minds if they are ready to learn their ropes.

\section{REFERENCES}

1. Tigerstedt R, Bergman PG. Niere und Kreislauf. Scand Arch Physiol. 1898; 8: 223.

2. Unger T, Paulis L, Sica DA. Therapeutic perspectives in hypertension: novel means for renin-angiotensinaldosterone system modulation and emerging devicebased approaches. Eur Heart J. 2011 Nov;32(22):2739-47. doi: 10.1093/eurheartj/ehr253.

3. Fraga-Silva RA, Ferreira AJ, Dos Santos RA. Opportunities for targeting the angiotensin-converting enzyme 2/angiotensin-(1-7)/mas receptor pathway in hypertension. Curr Hypertens Rep. 2013 Feb;15(1):31-8. doi: 10.1007/s11906-012-0324-1. PMID: 23212695. 